\title{
OPTIMAL OUTPUT FEEDBACK CONTROL: A MULTI-MODEL APPROACH
}

\author{
A. Varga \\ DLR - Oberpfaffenhofen \\ German Aerospace Research Establishment \\ Institute for Robotics and System Dynamics \\ P.O.B. 1116, D-82230 Wessling, Germany \\ Andreas.Varga@dlr.de
}

\begin{abstract}
In this paper we describe a computational approach to the optimal output feedback control of multi-model systems by means of a unique constant output feedback matrix. Extension of the method to output feedback control of multi-model periodic systems is also presented. The power of the proposed approach is illustrated by the simultaneous stabilization and optimization of a helicopter multi-model.
\end{abstract}

\section{Introduction}

Dynamical system models of plants operating around particular operating conditions are often obtained as multi-model representations of the form

$$
\begin{aligned}
\lambda x(t) & =A_{i} x(t)+B_{i} u(t) \\
y(t) & =C_{i} x(t)+D_{i} u(t)
\end{aligned},
$$

where $x(t) \in \mathbb{R}^{n}, u(t) \in \mathbb{R}^{m}, y(t) \in \mathbb{R}^{p} ; A_{i}, B_{i}, C_{i}$, $D_{i}$, for $i=1, \ldots, N$ are real matrices of appropriate dimensions; and where $\lambda x(t)=\dot{x}(t)$ for a continuous-time system or $\lambda x(t)=x(t+1)$ for a discrete-time system. The multi-model (1) can be alternatively specified by the sequence of system matrices

$$
S_{i}=\left[\begin{array}{cc}
A_{i} & B_{i} \\
C_{i} & D_{i}
\end{array}\right], \quad i=1, \ldots, N
$$

Such a model belongs to the more general class of socalled polytopic models

$$
\begin{aligned}
\lambda x(t) & =A(t) x(t)+B(t) u(t) \\
y(t) & =C(t) x(t)+D(t) u(t)
\end{aligned}
$$

whose time-varying system matrix $S(t)=\left[\begin{array}{ll}A(t) & B(t) \\ C(t) & D(t)\end{array}\right]$ varies within a fixed polytope of matrices

$S(t) \in \operatorname{Co}\left\{S_{1}, \ldots, S_{N}\right\}:=\left\{\sum_{i=1}^{N} \alpha_{i} S_{i}: \alpha_{i} \geq 0, \sum_{i=1}^{N} \alpha_{i}=1\right\}$.
The systems $S_{1}, \ldots, S_{N}$ are called vertex systems.

Let $J$ be a quadratic performance index of the form

$$
J=E\left\{\int_{0}^{\infty}\left[x(t)^{T} Q x(t)+u(t)^{T} R u(t)\right] d t\right\}
$$

in the continuous-time case, or

$$
J=E\left\{\sum_{k=0}^{\infty}\left[x(t)^{T} Q x(t)+u(t)^{T} R u(t)\right]\right\}
$$

in the discrete-time case, where $Q$ and $R$ are symmetric matrices with $Q \geq 0$ and $R>0$. In this paper we address the problem to determine the optimal outputfeedback control law

$$
u^{*}(t)=F y(t)
$$

which, according to the type of the system, minimizes either the performance index (3) or (4), and simultaneously stabilizes all $N$ systems in (1). In what follows we assume that such a matrix exists.

The simultaneous stabilization by output feedback is not a convex problem, thus techniques based on solving linear matrix inequalities (LMIs) [1] are not directly applicable to solve this problem. The solution which we propose relies on using gradient search techniques to find only a local minima of a modified performance index. The computational approach for the optimal output feedback control of a multi-model system is solved by turning the original problem into a large order highly structured standard output feedback problem with a modified performance index. Explicit expressions for evaluating the modified function and its gradient are derived. These computations involve successive solution of low order Lyapunov equations which can be done in parallel. The proposed approach is illustrated on a 24 th order helicopter multi-model with 41 vertex systems. Extension of the proposed approach to solve the periodic output feedback control of multi-model periodic systems is also discussed. 


\section{Single-Model Optimal Output Feedback Control}

Let us first review some results for the standard case of a single model (see also $[2,3]$ )

$$
\begin{aligned}
\lambda x(t) & =A x(t)+B u(t) \\
y(t) & =C x(t)+D u(t)
\end{aligned},
$$

for which we want to determine an optimal output feedback controller of the form (5) which minimizes either the performance index (3) or (4), according to the type of the system. Without loss of generality we assume $D=0$. It is easy to see that if $D \neq 0$ and $\widetilde{F}$ is the solution of the above problem for $D$ set to zero, then the solution for nonzero $D$ is $F=(I+\widetilde{F} D)^{-1} \widetilde{F}$, provided $I+\widetilde{F} D$ is invertible.

To solve the optimal output feedback problem descent methods based on gradient search techniques are usually employed. This approach involves the evaluation of the performance index and its gradient for a given stabilizing value of the output gain matrix $F$. We assume that the initial state at $t=0$ is a random variable with covariance matrix $E\left\{x(0) x^{T}(0)\right\}=G$. We denote in what follows $\bar{A}=A+B K C$ and $\bar{Q}=Q+C^{T} F^{T} R F C$. With these notations the values of function and its gradient can be computed for a continuous-time system as

$$
\begin{aligned}
J(F) & =\operatorname{tr}(P G) \\
\nabla_{F} J(F) & =2\left(B^{T} P+R F C\right) S C^{T},
\end{aligned}
$$

where $P$ and $S$ satisfies the continuous-time Lyapunov equations

$$
\begin{aligned}
\bar{A}^{T} P+P \bar{A}+\bar{Q} & =0 \\
\bar{A} S+S \bar{A}^{T}+G & =0 .
\end{aligned}
$$

The corresponding expressions for a discrete-time system are

$$
\begin{aligned}
J(F) & =\operatorname{tr}(P G) \\
\nabla_{F} J(F) & =2\left(B^{T} P \bar{A}+R F C\right) S C^{T}
\end{aligned}
$$

and $P$ and $S$ satisfies the discrete-time Lyapunov equations

$$
\begin{aligned}
P & =\bar{A}^{T} P \bar{A}+\bar{Q} \\
S & =\bar{A} S \bar{A}^{T}+G
\end{aligned}
$$

Having explicit analytical expressions for the function and its gradient it is easy to employ any gradient based technique to minimize $J$, provided an initial stabilizing output feedback gain is available.

An important aspect for what follows is to cope with structured feedback gain matrices. How structure enters in computations has been shown in [4] in the context of optimal decentralized control. By assuming that some elements of $F$ are fixed (for instance set to zero), we can solve the output feedback optimization problem by using straightforward mapping mechanisms to extract the active set of components of $F$ and of the gradient $\nabla_{F} J(F)$ before employing them by the minimization routine. Notice that although the parameter search is performed on a reduced set of elements of $F$, the above expressions of function and gradient are still valid.

As mentioned before, to use gradient search methods, an important subproblem in solving an optimal output feedback control problem is the initialization of the search process in the case of a given unstable system. An initial stabilizing output feedback gain $F$ can be computed by several search techniques [2]. Probably the simplest and most convenient approach is to use the minimization procedure itself to find a stabilizing feedback. This can be done by solving a sequence of modified problems which finally lead to a stabilizing gain matrix if one exists. For instance in the continuous-time case we can solve the optimal output feedback problem repeatedly for systems with $A$ replaced by $A+\alpha_{j} I$, $j=1,2, \ldots$, to compute the corresponding $F_{j}$, where $F_{0}=0$ and the strictly increasing sequence $\left\{\alpha_{j}\right\}$ is chosen such that $\operatorname{Re} \lambda_{i}\left(A+B F_{j-1} C+\alpha_{j} I\right)<0$. A stabilizing output gain has been found when $\alpha_{j} \geq 0$ at a certain moment. This technique can be even used to achieve eventually a prescribed stability degree for the closedloop system. A similar approach can be employed for stabilizing of a discrete-time system. This time we replace the pair $(A, B)$ with $\left(\alpha_{j} A, \alpha_{j} B\right)$ to compute the corresponding optimal $F_{j}$, where as before $F_{0}=0$ and the strictly increasing sequence $\left\{\alpha_{j}\right\}$ is chosen such that $\alpha_{j}\left|\lambda_{i}\left(A+B F_{j-1} C\right)\right|<1$. A stabilizing output gain has been found when $\alpha_{j} \geq 1$ at a certain moment.

\section{Multi-Model Optimal Output Feedback Control}

Consider now the optimal output feedback problem formulated in the Introduction. For the following developments we assume then for all vertex systems $D_{i}=D$, for $i=1, \ldots, N$, that is the feedthrough matrix is a constant matrix. Moreover without further loss of generality we can also assume $D=0$. We consider the following large system consisting of $N$ subsystems

$$
\begin{aligned}
\lambda x_{i}(t) & =A_{i} x_{i}(t)+B_{i} u_{i}(t), \quad i=1, \ldots, N, \\
y_{i}(t) & =C_{i} x_{i}(t)
\end{aligned}
$$

where $x_{i}(t) \in \mathbb{R}^{n}, u_{i}(t) \in \mathbb{R}^{m}, y_{i}(t) \in \mathbb{R}^{p}$. The overall system is described by

$$
\begin{aligned}
\lambda x(t) & =A x(t)+B u(t) \\
y(t) & =C x(t)
\end{aligned}
$$

with $x(t) \in \mathbb{R}^{n N}, u(t) \in \mathbb{R}^{m N}, y(t) \in \mathbb{R}^{p N}$ and the matrices $A=\operatorname{diag}\left(A_{1}, \ldots, A_{N}\right), B=\operatorname{diag}\left(B_{1}, \ldots, B_{N}\right)$, $C=\operatorname{diag}\left(C_{1}, \ldots, C_{N}\right)$. We consider the decentralized 
linear control law of the form

$$
u_{i}(t)=F_{i} y_{i}(t), \quad i=1, \ldots, N
$$

and we introduce the block-diagonal feedback gain ma$\operatorname{trix} F=\operatorname{diag}\left(F_{1}, \ldots, F_{N}\right)$ for the overall system (12). We define also the corresponding performance indices (3) or (4), where $Q=\operatorname{diag}\left(\beta_{1} Q, \ldots, \beta_{N} Q\right)$ and $R=$ $\operatorname{diag}\left(\beta_{1} R, \ldots, \beta_{N} R\right)$ and $\beta_{i}$ are weightings satisfying $\sum_{i=1}^{N} \beta_{i}=1$. By starting with the expressions for the decentralized output feedback derived in [4], it can be shown that for a given set of gain matrices $\left\{F_{i}\right\}$ in the continuous-time case the performance index and its gradient can be computed for $i=1, \ldots, N$ as

$$
\begin{aligned}
J\left(\left\{F_{i}\right\}\right) & =\sum_{i=1}^{N} \beta_{i} \operatorname{tr}\left(P_{i} G\right) \\
\nabla_{F_{i}} J\left(\left\{F_{i}\right\}\right) & =2 \beta_{i}\left(B_{i}^{T} P_{i}+R_{i} F_{i} C_{i}\right) S_{i} C_{i}^{T},
\end{aligned}
$$

where $P_{i}$ and $S_{i}$ satisfy the following continuous-time Lyapunov equations

$$
\begin{array}{r}
\bar{A}_{i}^{T} P_{i}+P_{i} \bar{A}_{i}+\bar{Q}_{i}=0 \\
\bar{A}_{i} S_{i}+S_{i} \bar{A}_{i}^{T}+G=0
\end{array}
$$

with $\bar{A}_{i}=A_{i}+B_{i} F_{i} C_{i}, \bar{Q}_{i}=Q+C_{i}^{T} F_{i}^{T} R F_{i} C_{i}$ and $G=E\left\{x(0) x^{T}(0)\right\}$. The corresponding expressions for a discrete-time system are

$$
\begin{aligned}
J\left(\left\{F_{i}\right\}\right) & =\sum_{i=1}^{N} \beta_{i} \operatorname{tr}\left(P_{i} G\right) \\
\nabla_{F_{i}} J\left(\left\{F_{i}\right\}\right) & =2 \beta_{i}\left(B_{i}^{T} P_{i} \bar{A}_{i}+R F_{i} C_{i}\right) S_{i} C_{i}^{T},
\end{aligned}
$$

and $P_{i}$ and $S_{i}$ satisfy the following discrete-time Lyapunov equations

$$
\begin{aligned}
P_{i} & =\bar{A}_{i}^{T} P_{i} \bar{A}_{i}+\overline{Q_{i}} \\
S_{i} & =\bar{A}_{i} S_{i} \bar{A}_{i}^{T}+G .
\end{aligned}
$$

By imposing that all feedback components are equal $F_{1}=\cdots=F_{N}=F$ we further obtain

$$
\begin{aligned}
J(F) & =\sum_{i=1}^{N} \beta_{i} \operatorname{tr}\left(P_{i} G\right) \\
\nabla_{F} J(F) & =2 \sum_{i=1}^{N} \beta_{i}\left(B_{i}^{T} P_{i}+R F C_{i}\right) S_{i} C_{i}^{T}
\end{aligned}
$$

for a continuous-time system and

$$
\begin{aligned}
J(F) & =\sum_{i=1}^{N} \beta_{i} \operatorname{tr}\left(P_{i} G\right) \\
\nabla_{F} J(F) & =2 \sum_{i=1}^{N} \beta_{i}\left(B_{i}^{T} P_{i} \bar{A}_{i}+R F C_{i}\right) S_{i} C_{i}^{T}
\end{aligned}
$$

for a discrete-time system, where $\bar{A}_{i}=A_{i}+B_{i} F C_{i}$. The matrices $P_{i}$ and $S_{i}$, according to the type of the system, satisfy the Lyapunov equations (13) or (14), where $\bar{Q}_{i}=Q+C_{i}^{T} F^{T} R F C_{i}$.

It is apparent that the evaluation of function and gradient values implies the independent computation of $N$ function and gradient values for each vertex subsystem. This can be done in parallel and thus the evaluation of function and gradient is potentially very efficient on parallel architecture machines.
The use of weights is a convenient means to experiment with sub-collection of systems. Weights can be also useful to put more emphasis on the performances of some particular instances of parameter values (for instance on nominal values).

In the case of a polytopic model, even if a particular optimization led to a stabilizing output feedback matrix for all vertex systems, there is still no guaranty that the polytopic system is stable, that is any system in the convex hull Co $\left\{S_{1}, \ldots, S_{N}\right\}$ is stable for the computed $F$. In order to check the stability in this case we can try to solve simultaneously for $i=1, \ldots, N$ the following LMIs of the form

$$
\begin{aligned}
\left(A_{i}+B_{i} F C_{i}\right) X+X\left(A_{i}+B_{i} F C_{i}\right)^{T} & <0 \\
X & >0
\end{aligned}
$$

in continuous-time case or

$$
\begin{array}{r}
\left(A_{i}+B_{i} F C_{i}\right) X\left(A_{i}+B_{i} F C_{i}\right)^{T}-X<0 \\
X>0
\end{array}
$$

in the discrete-time case. Notice that if the above stability check is positive, then the polytopic model is stable for arbitrarily fast time variations within the convex hull, which indicates a certain conservatism of such kind of results.

Having analytical expressions for function and gradients, we can employ in principle any gradient based minimization technique to solve the optimization problem. It is however advisable to use search methods where the user has some control on choosing the step lengths because too large step lengths can lead to feedback gains which destabilize one or more vertex systems and thus can lead to failures because of unavailability of function and gradient values. An example of a method well suited for complex optimization purposes is the limited memory BFGS method [5] using the line search procedure with guaranteed decrease described in [6]. Both methods are implemented within the MINPACK2 project ( the successor of MINPACK-1 [7]) offering a convenient reverse communication interface which allows a complete separation between the optimization routines and the routines implementing the complex computations for function and gradient evaluations.

\section{Multi-Model Optimal Periodic Output Feedback Control}

The approach presented in the previous section can be readily extended to the case of periodic multi-models. Consider the periodic discrete-time systems

$$
\begin{aligned}
x(t+1) & =A_{i}(t) x(t)+B_{i}(t) u(t) \\
y(t) & =C_{i}(t) x(t)
\end{aligned}
$$

where $A_{i}(t)=A_{i}(t+K), B_{i}(t)=B_{i}(t+K), C_{i}(t)=$ $C_{i}(t+K)$ for $i=1, \ldots, N$ are periodic real matrices of 
period $K$. We want to compute a periodic output feedback matrix $F(t)$ such that the output feedback control law

$$
y(t)=F(t) u(t)
$$

stabilizes simultaneously all $N$ periodic systems (15) and minimizes the performance index

$$
J=E\left\{\sum_{k=0}^{\infty}\left[x(t)^{T} Q(t) x(t)+u(t)^{T} R(t) u(t)\right]\right\},
$$

where $Q(t)$ and $R(t)$ are periodic symmetric nonnegative definite matrices.

To simplify notations we introduce the script notation $\mathcal{X}$ which associates the block-diagonal matrix

$$
\mathcal{X}=\operatorname{diag}(X(0), X(1), \ldots, X(K-1))
$$

to the cyclic sequence of matrices $X(t), t=0, \ldots, K-1$. This notation is consistent with the standard matrix operations. We denote with $\sigma \mathcal{X}$ the $K$-cyclic shift

$$
\sigma \mathcal{X}=\operatorname{diag}(X(1), \ldots, X(K-1), X(0))
$$

applied to the cyclic sequence $X(t), t=0, \ldots, K-1$.

By using a similar approach as in the previous section, we can derive the expressions of the function and gradient by using the results of [8]. For each vertex system the computations involve the solution of two discrete periodic Lyapunov equations: one in reverse time

$$
\mathcal{P}_{i}=\overline{\mathcal{A}}_{i}^{T} \sigma \mathcal{P}_{i} \overline{\mathcal{A}}_{i}+\overline{\mathcal{Q}}_{i}
$$

and one in forward time

$$
\sigma \mathcal{S}_{i}=\overline{\mathcal{A}}_{i} \mathcal{S}_{i} \overline{\mathcal{A}}_{i}^{T}+\mathcal{G}
$$

where $\overline{\mathcal{A}}_{i}=\mathcal{A}_{i}+\mathcal{B}_{i} \mathcal{F} \mathcal{C}_{i}, \overline{\mathcal{Q}}_{i}=\mathcal{Q}+\mathcal{C}_{i}^{T} \mathcal{F}^{T} \mathcal{R} \mathcal{F} \mathcal{C}_{i}$, and $\mathcal{G}=\operatorname{diag}\left(0,0, \ldots, E\left\{x(0) x^{T}(0)\right\}\right)$. For the expression of the function and its gradient we obtain

$$
\begin{aligned}
J(\mathcal{F}) & =\sum_{i=1}^{N} \beta_{i} \operatorname{tr}\left(\sigma \mathcal{P}_{i} \mathcal{G}\right) \\
\nabla_{\mathcal{F}} J(\mathcal{F}) & =2 \sum_{i=1}^{N} \beta_{i}\left(\mathcal{B}_{i}^{T} \sigma \mathcal{P}_{i} \overline{\mathcal{A}}_{i}+\mathcal{R} \mathcal{F} \mathcal{C}_{i}\right) \mathcal{S}_{i} \mathcal{C}_{i}^{T}
\end{aligned}
$$

Efficient algorithms to solve the discrete periodic Lyapunov equations (17) and (18) have been recently developed in [9] and can be used to evaluate efficiently the above expressions for function and gradient.

To check the stability we can try to solve simultaneously for $i=1, \ldots, N$ the following LMIs

$$
\begin{array}{r}
\left(\mathcal{A}_{i}+\mathcal{B}_{i} \mathcal{F C}_{i}\right) \mathcal{X}\left(\mathcal{A}_{i}+\mathcal{B}_{i} \mathcal{F C}_{i}\right)^{T}-\sigma \mathcal{X} \\
\mathcal{X}>0
\end{array}
$$

If a solution to the above LMIs exits then the periodic output feedback $\mathcal{F}$ is stabilizing over the convex hull of all periodic vertex systems.
It is interesting to explore the possibility to stabilize constant multi-model systems by using time-varying periodic output feedback gains. A trivial necessary condition that the optimal multi-model output feedback problem has a solution for a time-invariant multi-model system of the form (1) is that there exists a stabilizing output feedback gain which simultaneously stabilizes all vertex systems. It is a well know fact that not all systems are stabilizable by output feedback thus the approach proposed in Section 3 is not generally applicable. However, analogously as in the single model case [8] we can try to employ periodic output feedback for the control of constant systems which are not output stabilizable. We conjecture that provided the period $K$ is chosen sufficiently high every constant multi-model system can be stabilized by using periodic output feedback. Moreover, each multi-model system can not only be stabilized by the periodic output feedback but even an arbitrary fast dynamics can be achieved simultaneously for all vertex systems. Thus the periodic output feedback could be a very promising approach for practical multi-model applications.

\section{Application to Helicopter Ground Resonance Stabilization}

Due to the wide range of helicopter operating conditions, payload configurations, and flight regimes, several specific helicopter control tasks, as for instance ground or air resonance stabilization [10, 11], lead to a natural multi-model formulation of the underlying control problem. To show the potential of the proposed multi-model approach for output feedback control, we consider the active control for augmenting rotor/body damping and to improve aeromechanical stability in case of the ground resonance problem. The active control could become a viable cheap alternative to the use passive technique based on mechanical or elastomeric blade dampers.

The nonlinear dynamics of a helicopter on ground over a wide range of rotor speeds and thrust values can be alternatively described as a multi-model linear system of the form (1), where each vertex system corresponds to a pair of rotor-speed-thrust values $(\Omega, T)$. For numerical experimentations we employed models obtained with the help of CAMRAD/JA software for a generic helicopter model for 31 rotor speed values ranging from $80 \%$ to $110 \%$ of the nominal rotor speed $\Omega_{0}$ and for 11 thrust values at $98 \% \Omega_{0}$ (the rotor speed for ground resonance) ranging from 0 to $100 \%$ of the maximum thrust $\left(T_{\max }\right)$. The system has two control inputs, four measurable outputs and 24 state variables. The dynamics of each model includes also two first order models of actuators and two second order low pass filters. The filters are intended to limit the control effects to a frequency domain around and bellow the resonance frequency (about 

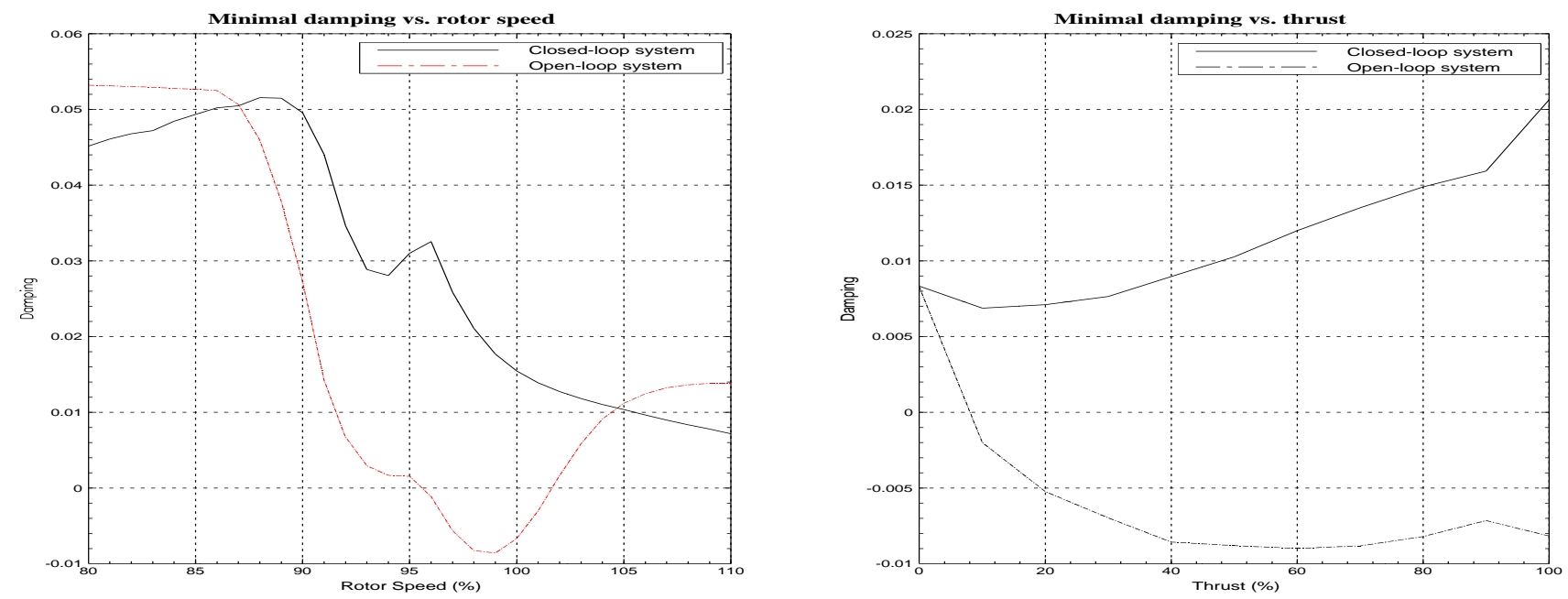

Figure 1: Damping vs. rotor speed and thrust (uniform performance weighting)
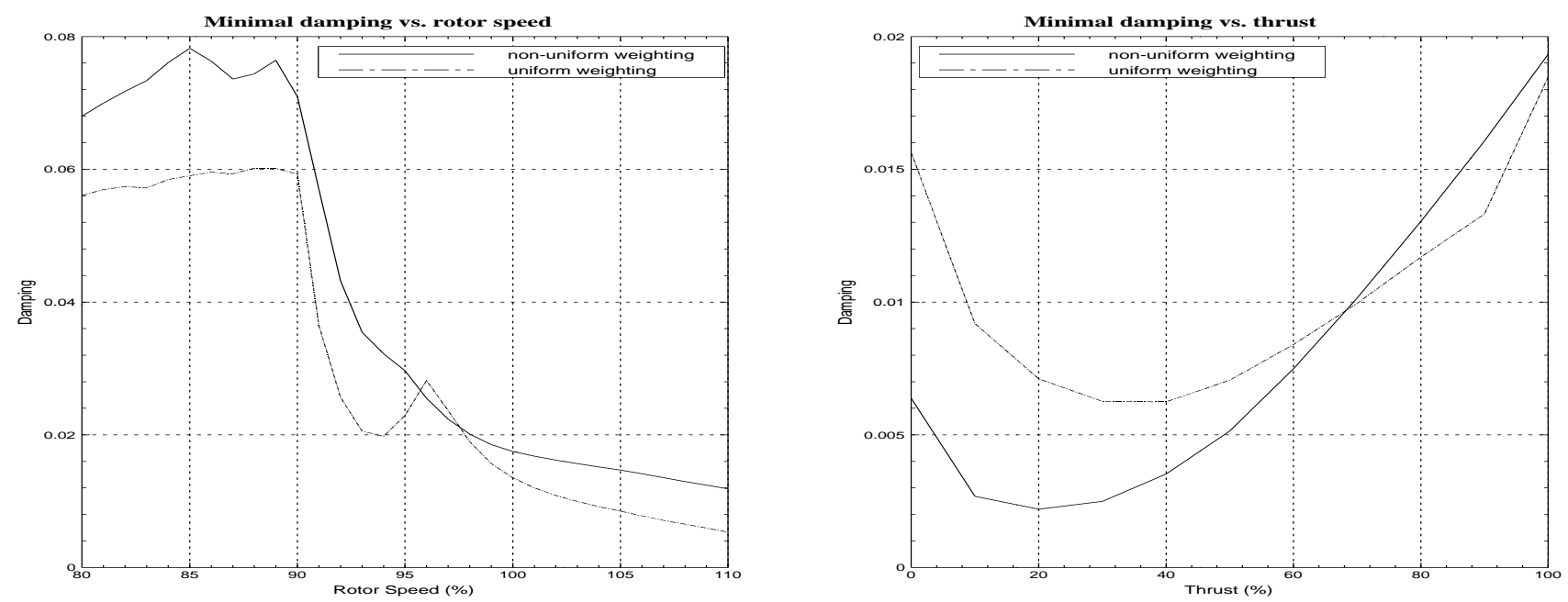

Figure 2: Damping vs. rotor speed and thrust (non-uniform performance weighting)

$10 \mathrm{~Hz}$ ). The plots of values of the minimum damping for frequencies under $10 \mathrm{~Hz}$ over all rotor speeds and thrust values show the essential aeromechanical instability of the open loop helicopter dynamics in the region around the $98 \% \Omega_{0}$ virtually for all thrust values (see Figure 1).

The control problem consists in determining, if possible, a single output feedback controller which is able to suppress the ground resonance at all rotor speeds and for all thrust values and produce an augmentation of rotor/body damping to values which correspond to those usually obtained by using passive techniques. The feasibility of designing a unique controller which stabilizes all systems over all rotor speeds at nominal thrust was evidenced in $[11,12]$, by using a carefully designed controller for the $\left(98 \% \Omega_{0}, T_{\max }\right)$ model. By averaging all controllers over all rotor speeds and thrust values it was possible even to stabilize all vertex systems [12]. Although these techniques are rather heuristic, they rise the question of existence of a systematic approach to solve the simultaneous stabilization and optimization problem. Such an approach is precisely that proposed in this paper.

As it could be expected, in order to stabilize all systems, it is enough to stabilize a representative subsets of models. The stabilization of all models was achieved by using only 7 models (for 5 rotor speeds: $80 \% \Omega_{0}$, $90 \% \Omega_{0}, 98 \% \Omega_{0}, 104 \% \Omega_{0}, 110 \% \Omega_{0}$, and 2 thrust values: $30 \% T_{\max }, 50 \% T_{\max }$ ) from a total of 41 distinct available models. The curves for minimal damping in the open-loop and the closed-loop systems for uniformly weighted performance indexes $\left(\beta_{i}=1 / N\right)$ are shown in Figure 1. By adding more models the interpolation properties of the resulting controller are usually better. The choice of models to be added can be done by looking at the non-smooth parts on the curves. By adding just those models where the non-smooth behav- 
ior manifests the damping characteristic can be locally improved leading finally to a satisfactory smooth global characteristic.

Another aspect studied was the use of non-uniform weightings of the individual performance indexes. Although it was shown that it is possible to stabilize all systems with a unique controller, the damping properties shown in Figure 1 are unsatisfactory for rotor speeds greater than $\Omega_{0}$. We can try to use a gain scheduling approach with respect to thrust in the hope that for each thrust value we can achieve a satisfactory damping over all corresponding rotor speed values. For such an approach, by means of appropriately chosen weightings, we can put more emphasis on enforcing better performance over all rotor speed values for a given thrust than on the simultaneous stabilization over the rest of thrust values. The stabilization over all thrust values is still necessary in order to guarantee the global stability of each controller. In Figure 2 are shown the results obtained by using a non-uniform weighting to optimize the performance at maximum thrust. For the multimodel output feedback optimization we used 15 vertex systems. For comparison purpose the values for uniform weighting for the same set of models are also presented. It can be seen that the damping performance over all rotor speed values is significantly better than that obtained by using a uniform weighting but the values over the thrust values are sensibly worse at low thrust. A similar behavior is to be expected for performance optimization at other thrust values. It seems that this behavior is an intrinsic limitation of the helicopter dynamics. A possibility to be further investigated is the use of alternative filters or of alternative higher order output feedback controllers.

\section{Conclusions}

We described a possible computational approach based on nonlinear programming techniques for the simultaneous stabilization and optimization of a multi-model system. The proposed approach has been extended to solve a similar problem for multi-model discrete periodic systems. The new multi-model optimization technique was employed to derive a systematic approach for the stabilization of non-linear helicopter models over a wide range of rotor speed and thrust values. The provision to achieve increased performance for particular subset of model was demonstrated by the use of non-uniform performance weightings. LAPACK [13] based FORTRAN 77 routines to evaluate function and gradient for the proposed multi-model output feedback optimization approach have been implemented. A versatile interactive user interface for this approach has been developed as a computational module within the computer aided control engineering environment ANDECS of DLR. This module allows an easy manipulation of multi-model sys- tems via a specialized database and a simple, straightforward operation to perform optimization studies. Several supplementary features (prescribed stability degree, structured feedback) add useful operational facilities.

\section{References}

[1] S. Boyd, L. El Ghaoui, E. Feron, and V. Balakrishnan. Linear Matrix Inequalities in System and Control Theory. SIAM, 1994.

[2] P. M. Mäkilä and H. T. Toivonen. Computational methods for parametric LQ problems - a survey. IEEE Trans. Autom. Control, 32:658-671, 1987.

[3] S. Pieters. Gradient methods for the computation of the LQ optimal output feedback. Technical Report TR R186-95, DLR-Oberpfaffenhofen, German Aerospace Research Establishment, Institute for Robotics and System Dynamics, April 1995.

[4] H. T. Toivonen and P. M. Mäkilä. A descent Anderson-Moore algorithm for optimal decentralized control. Automatica, 21:743-744, 1985.

[5] D. C. Liu and J. Nocedal. On the limited memory BFGS method for large scale optimization. Mathematical Programming, 45:503-528, 1989.

[6] J. J. Moré and D. J. Thuente. On line search algorithms with guaranteed sufficient decrease. Mathematics and Computer Science Division Preprint MCSP330-1092, Argonne National Laboratory, Argone, IL, October 1990.

[7] J. J. Moré. User's Guide for MINPACK-1. Applied Mathematics Division Report ANL-80-74, Argonne National Laboratory, Argone,IL, 1980.

[8] A. Varga and S. Pieters. A computational approach for optimal periodic output feedback control. In Proc. CACSD'96 Symposium, Dearborn, MI, 1996.

[9] A. Varga. Periodic Lyapunov equations: some applications and new algorithms. Technical Report TR R197-96, DLR-Oberpfaffenhofen, Institute for Robotics and System Dynamics, February 1996. (submitted for publication).

[10] D. Teves, G. Niesl, A. Blaas, and S. Jacklin. The role of rotor active control in future rotorcraft. In Proc. 21st European Rotorcraft Forum, St. Petersburg, volume III, pages 10.1-10.17, 1995.

[11] Ch. Kessler and G. Reichert. Active control of ground and air resonance including transition from ground to air. In Proc. 20th European Rotorcraft Forum, Amsterdam, pages 64.1-64.17, 1994.

[12] D. Teves. (private communication). 1996.

[13] E. Anderson, Z. Bai, J. Bishop, J. Demmel, J. Du Croz, A. Greenbaum, S. Hammarling, A. McKenney, S. Ostrouchov, and D. Sorensen. LAPACK User's Guide, Second Edition. SIAM, Philadelphia, 1995. 